\title{
Diagnosis And Monitoring Of Chronic Kidney Disease Progression In Patients With Diabetes Mellitus - A Retrospective Study
}

${ }^{1}$ Department of Diabetology, Clinical County Emergency Hospital Constanța

2Department of Pathology , Faculty of Medicine, University „Ovidius” Constanța

ABSTRACT. The study was retrospective and the purpose was to assess how screening and monitoring of chronic kidney disease are performed in patients with diabetes mellitus, in terms of routine medical practice. The study was performed on a sample of 127 patients with diabetes mellitus who died during hospitalization, between 2006-2010. Data were collected from autopsy reports and from clinical medical records of patients with diabetes mellitus, aiming for 5 years prior to death. The main cause of death was represented by cardiovascular events (61.4\% of patients). Most of the patients associate high blood pressure and dyslipidemia. To diagnosis and monitoring the development of chronic kidney disease were performed albuminuria measurements ( 0.6 determinations / patient / year) and serum creatinine measurements $(0.41$ determinations $/$ patient / year). $91.8 \%$ of patients with diabetes duration greater than 1 year had at least one determination of albuminuria within five years. Most patients in the study group associated multiple cardiovascular risk factors. Investigations for diagnosis and monitoring the development of diabetic kidney disease are performed less frequently than recommendations and the causes are multiple.

Key words: diabetic kidney disease, chronic kidney disease, albuminuria, diabetes mellitus

\section{Introduction}

In the last years, there has been a marked increase in cases of diabetes mellitus, particularly type 2 diabetes [1]). Diabetic kidney disease is one of the microvascular complications of diabetes with important consequences on morbidity and mortality [2]. Diagnosis of diabetic kidney disease is based on urinary excretion of albumin and glomerular filtration rate measurement and should be done earlier in order to treat and slow the progression to end stage renal disease.

Microalbuminuria is the first manifestation of glomerular damage in type 1 diabetes mellitus. Macroalbuminuria is a characteristic of patent diabetic kidney disease. In type 2 diabetes mellitus, microalbuminuria is not only a marker of diabetic nephropathy, but also a marker of cardiovascular disease, a predictor of coronary artery disease, peripheral vascular disease and cardiovascular mortality.

In type 1 diabetes mellitus, most patients who develop diabetic nephropathy will progress to the progressive decline in renal function [3]. End stage renal disease is the cause of death of over $50 \%$ of patients with type 1 diabetes and diabetic kidney disease. In type 2 diabetes, the rate of decline of renal function is varied from one patient to another. Increased risk of death from coronary heart disease in elderly patients with type 2 diabetes prevent many patients with diabetes and chronic kidney disease to evolve to end stage renal disease. Risk factors for progression to chronic renal disease are represented by diabetes duration, metabolic control, association of hypertension, hyperuricemia, obesity $[4,5]$, 
dyslipidemia [6], male gender and smoking [7].

The study aims to evaluate how screening and monitoring of chronic kidney disease are done in diabetic patients in routine medical practice.

\section{Materials and Methods}

The study was retrospective and was conducted on a sample of 127 patients with diabetes mellitus who died during hospitalization in Clinical County Emergency Hospital Constanta, between 2006-2010, which autopsies were performed in the Pathology Department. A large proportion of patients in the study group showed at autopsy kidney damage secondary to diabetes, hypertension and dyslipidemia. This study aimed to assess the frequency of investigations carried out in clinical practice to assess risk factors, to diagnose and monitoring the development of chronic kidney disease in patients with diabetes. Data about causes of death, duration of diabetes, comorbidities, laboratory investigations were collected from autopsy reports and from clinical medical records of patients with diabetes, aiming for 5 years prior to death.

\section{Results and Discussions}

The structure of the study group was examined according to the distribution by age, sex, urban or rural areas, type of diabetes mellitus, causes of death.

Distribution by age of patients was as follows: 0 patients younger than 20 years, 4 patients (3.1\%) aged $20-40$ years, 24 patients $(18.9 \%)$ aged 40-60 years, 90 patients $(70.9 \%)$ aged $60-80$ years, 9 patients $(7.1 \%)$ older than 80 years.

In the study group were 69 women,

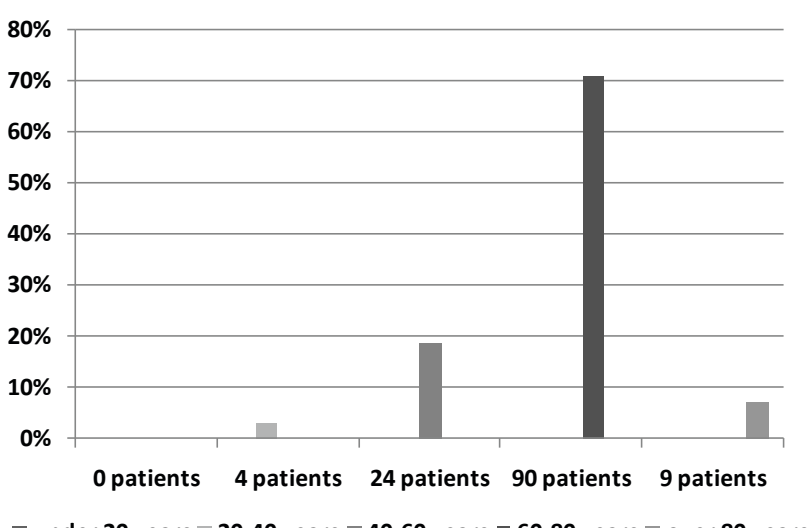

Figure 1 - Distribution by age groups

representing $54.3 \%$ and 58 men representing $45.7 \%$. 38 patients $(29.9 \%)$ came from rural areas and 89 patients $(70.1 \%)$ from urban areas. Distribution of patients according to type of diabetes mellitus was as follows: type 1 diabetes- 16 patients $(12.6 \%)$, type 2 diabetes-108 patients $(85.0 \%)$, secondary diabetes- 3 patients $(2.4 \%)$. Duration of diabetes mellitus ranged from 0 to 34 years: under 1 year 17 patients (13.4\%), between $1-10$ years 38 patients $(29.9 \%)$, between $10-20$ years 53 patients $(41.7 \%)$, over 20 years 19 patients $(15 \%)$.

Distribution of patients in relation to the cause of death was: cardiovascular events 78 patients

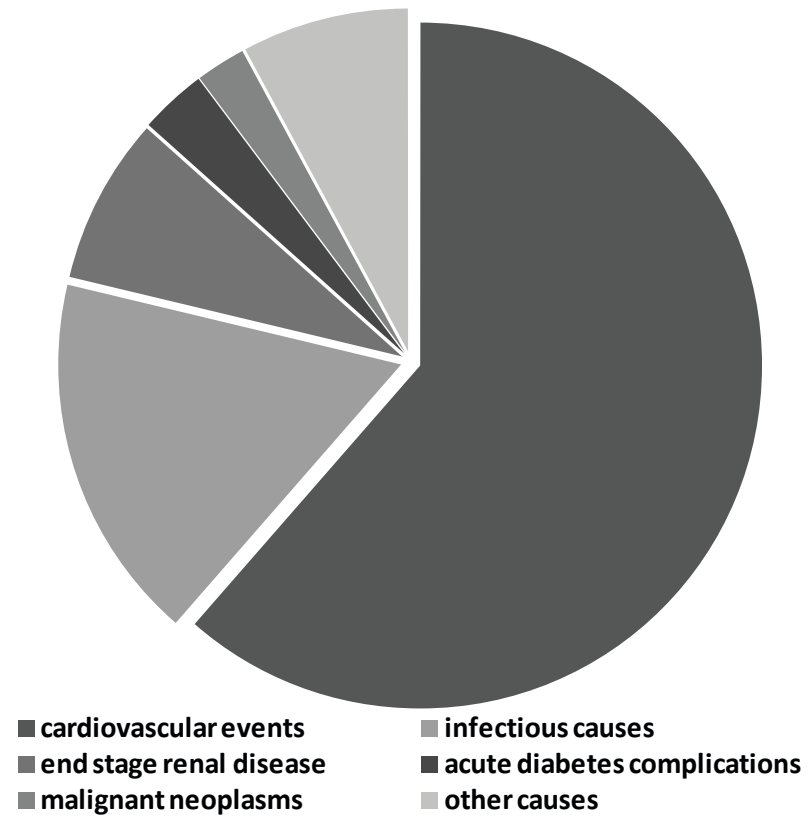

Figure 2 - Distribution by causes of death 


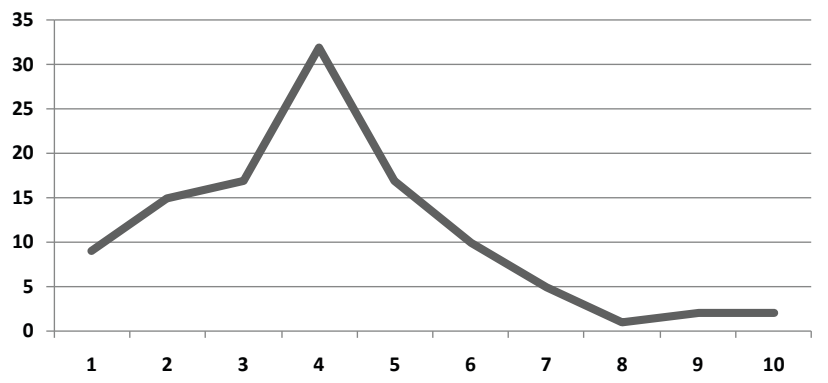

Figure 3 - Number of albuminuria measurements/ patient/ 5 years

(61.4\%), infectious causes 22 patients $(17.3 \%)$, end stage renal disease 10 patients $(7.9 \%)$, acute complications of diabetes mellitus 4 patients $(3.1 \%)$, malignant neoplasms 3 patients $(2.4 \%)$, other causes 10 patients $(7.9 \%)$.

The most frequent comorbidities encountered in the study group were hypertension (105 patients, representing $82.7 \%$ ) and coronary heart disease (78 patients, representing $61.4 \%$ ). Out of the 127 patients in the study group, 113 (88.9\%) were dyslipidaemics.

Screening for diabetic renal disease was performed by determining the urinary excretion of albumin and glomerular filtration rate assessment on the basis of serum creatinine, using the MDRD formula (Modification of Diet in Renal Disease), which takes into account age, race and sex.

A total of 17 patients had a duration of diabetes less than 1 year. The data about screening for diabetic kidney disease were analyzed in patients with diabetes duration greater than 1 year. Out of the 110 patients with diabetes duration of more than a year, albuminuria evaluation was performed in 101 patients (91.8\%) achieving a rate of 0.6 determinations/ patient/year, that means an investigation every 1.7 years. The investigations performed were: urinalysis, determination of the albumin / creatinine ratio in morning urine or dosing of urinary albumin excretion in nocturnal urine or in 24 hours urine. In patients diagnosed with diabetic kidney disease, albuminuria determinations were performed more frequently, achieving a rate of 1.42 determinations/patient/year and a determination at every 0.7 years.

Determination of serum creatinine, which allows measurement of glomerular filtration rate using the MDRD formula, was performed with a frequency of 0.47 determinations/patient/year, that means an investigation every 2.41 years. In patients diagnosed with diabetic kidney disease, determinations were made with a frequency of 1.2 determinations/patient/ year and a determination of serum creatinine every 0.8 years.

Blood glucose dosing frequency was difficult to assess because the study is retrospective, and a significant percentage of patients with diabetes mellitus had access to self monitoring of blood glucose at home. $\mathrm{HbA} 1 \mathrm{c}$ determination was performed with a frequency of 0.83 determinations/patient/year, namely a determination every 1.2 years. Other investigations performed were: urea, uric acid, lipid profile, blood count. Systolic and diastolic blood pressure and body weight were measured at each medical visit (every 3 or 6 months).

Investigations were recommended by the treating physician with a higher frequency, but were made in a lower percentage and the causes are related to increased costs of laboratory tests for patients. There were situations where no laboratory investigations were possible because patients were homebound or uncooperative.

\section{Conclusions}

Most patients in the study group were associated with multiple cardiovascular risk factors, as illustrated the largest share of cardiovascular diseases among causes of death $(61.4 \%$ of total). Investigations for diagnosis and monitoring the development of diabetic kidney disease are performed with a relatively low frequency ( 0.6 determinations/ patient/year for albuminuria, respectively 0.41 determinations/patient/year for serum creatinine) and the identified the causes are multiple.

Increased incidence of diabetes mellitus, particularly type 2 diabetes and increased survival of patients with diabetes due to development of the therapeutic and educational issues and to the self-monitoring blood glucose in diabetic patients increases the number of patients that may develop chronic complications, including diabetic kidney disease. Early detection, monitoring progress and appropriate therapy of diabetic kidney disease could 
slow its progression to end stage renal disease and death.

\section{References}

1. BOTEA V, SIMA A, ȘERBAN V. - Diabetul zaharat al vârstnicului. In Şerban V:Tratat român de boli metabolice, vol.1. Ed. Brumar, Timișoara, 2010: 461-472.

2. SERAFINCEANU C. - Boala renală diabetică. In Ionescu-Târgoviște C. Tratat de Diabet Paulescu. Ed. Academiei Române, Bucureşti, 2004, 897970.

3. MOGENSEN CE - Nephropathy and Hypertension in Diabetic Patients, in Belfiore F, Mogensen CE, eds: New Concepts in Diabetes and Its Treatment, Basel: Karger, 2000, 152-173.

4. WANG Y, CHEN X, SONG Y. - Association between obesity and kidney disease: a systematic review and meta-analysis. Kidney Int 2008; 73:19-33.

5. BALA C. - Riscurile obezității, complicații, comorbiditate. In Hâncu N, Roman G, Vereşiu I A. Diabetul zaharat, nutriția și bolile metabolice, Tratat 2. Editura Echinox, Cluj Napoca, 2010. 120-122.

6. MARTINEZ CASTELAO A. - Advances in diabetes mellitus, diabetic nephropathy, metabolic syndrome and cardio-vascular-renal risk. Nefrologia, 2008; Suppl 5:79-84.

7. AFGHANI H, CEDERHOLM J, ELIASSON B. Risk Factors for the Development of Albuminuria and Renal Impairment in Type 2 Diabetes. The Swedish National Diabetes Register. Nephrol Dial Transplant. 2011; 26(4): 1236-1243. 